\title{
Robust energy management system for multi-source DC energy systems - real-time setup and validation
}

\author{
Jean-Marc Nwesaty, Antoneta Iuliana Bratcu, Senior Member, IEEE, Alexandre Ravey, Member, IEEE, David Bouquain, \\ and Olivier Sename, Senior Member, IEEE
}

\begin{abstract}
This paper aims at providing a proof of concept of a systematically designed $\mathrm{LPV} / \mathcal{H}_{\infty}$-based energy management system (EMS) for coordinated multi-variable control of multi-source electrical systems. A three-source electrical system representing the power supply system on board of an electric vehicle has been chosen as a representative example of irregular and generally not a priori known load variation. The power supply system is composed of fuel cell, battery and supercapacitor. Each power source is coupled to a DCDC converter, all converters being connected in parallel to a common DC-bus in order to feed the load represented by the vehicle's electrical motor. The system is modelled as an LPV system - as its operating point depends on the load - and the control objectives are cast into the $\mathcal{H}_{\infty}$ formalism as a disturbance-rejection problem.

A dedicated hardware-in-the-loop system was built for proof of concept purpose, with real-world battery and supercapacitor being used, while the fuel cell system is entirely emulated. A dSPACE MicroAutoBox $^{\circledR}$ II device embeds the designed EMS, due to its flexibility and ease of programming with MATLAB ${ }^{\circledR}$. A driving cycle from IFSTTAR (Institut Français des Sciences et Technologies des Transports, de l'Aménagement et des Réseaux) is chosen as a pertinent scenario of load variation due to its rich frequency content able to challenge all the three sources. Effectiveness of the EMS is assessed in relation to the imposed control objectives - DC-bus voltage regulation, dynamical separation of power sources' current variations depending on the specialization range of each source, and imposing desired steady-state behavior for each of the three power sources - with very promising results.
\end{abstract}

Index Terms $-\mathcal{H}_{\infty}$ control, LPV systems, power source coordination, reduced-order controller, frequency separation, electric vehicle.

\section{INTRODUCTION}

$\mathbf{M}$ ULTI-SOURCE - also named hybrid - power supply systems are nowadays becoming ubiquitous, especially in applications where load variation is strongly irregular. The concept of "microgrid" has thus emerged, where the basic functional entities may operate either in the so-called "grid-connected" mode, or to supply power for a variety of stand-alone applications such as electrical mobility - or for islanded/isolated zones when they operate in "grid-forming" mode. Renewable energies, whose intermittency requires hybridization with storage units, may also be present in microgrids. In particular, DC microgrids have gained popularity vs. AC ones due to their more simplified operation (e.g., no need for synchronization). Stand-alone applications in avionic,

J.-M. Nwesaty, A. I. Bratcu and O. Sename are with Univ. Grenoble Alpes, CNRS, Grenoble INP*, GIPSA-lab, Control Systems Department, 38000 Grenoble, France (*Institute of Engineering). E-mails: nwesaty@gmail.com, antoneta.bratcu@gipsalab.fr, olivier.sename@gipsa-lab.fr.

A. Ravey and D. Bouquain are with FEMTO-ST Institute, Univ. Bourgogne Franche-Comté, UTBM, CNRS, Belfort, 90000, France. E-mails: alexandre.ravey@utbm.fr, david.bouquain@utbm.fr. automotive and marine industries, but also power supply of remote areas may benefit from this advantage, among others [1], [2].

Multi-source power supply systems use combinations of different storage technologies, such as fuel cells, batteries, supercapacitors, flying wheels, etc.. Ragone's plot [3], features two main classes of sources: high-energy-density sources that can supply power for long duration of time (e.g., fuel cells), and high-power-density sources, which can provide relatively high power for short period of time (e.g., supercapacitors). Batteries are classified in between the two classes depending on the battery type.

In the electromobility three main hybridization topologies are available depending on the number of components, energy management complexity and performance reliability: series, parallel, and cascaded [4]-[6]. In this paper, a parallel structure of three sources in a DC system - fuel cell, battery and supercapacitor - is chosen due to its flexibility (sources' independent operation, facility to replace/add power sources, etc.).

1) Related works: In multi-source systems an Energy Management System (EMS) should coordinate the different devices and ensure a desired power sharing between sources to satisfy the load demand, while preserving reliability of all sources and extending their remaining useful life [7].

Efficient EMS can be designed for a variety of applications. Model Predictive Control (MPC) - with its nonlinear and/or stochastic versions - may be applied at coordinated multivariable control strategies for stand-alone microgrids and/or multi-source systems, with or without renewable sources, as it successfully deals with multiple constraints [8]-[10]. But adequate plant mathematical modelling and also a priori knowledge of load variation to predict the behavior are needed. For electric vehicles, this results in drivingcycle-dependent solutions, which may be a disadvantage. Nonlinear methods have also been proposed, such as Lyapunov-based [11] or sliding mode [12]. More recently, interest of multi-agent-based approach and games theory has been revealed [13].

Complex techno-economical criteria must be considered in the multi-source EMS design. Thus, reliability-aware design rely on complex degradation and ageing models of storage units, along with optimization methods [14]. The multi-scale character of multisource systems justify hierarchical control structures [15], with dynamically separated layers for enabling operation of sources in their "specialization" range of variations - according to Ragone's taxonomy - to improve their reliability [16]. In this way, highpower-density sources (like ultracapacitors), specialized in providing high-amplitude fast-varying currents, are able to protect highenergy-density sources (like fuel cells or batteries), whose current 
variations must be smoother. Dynamic separation can be done by filtering, where variations of current of each source are confined in a predefined frequency interval [17]-[19]. The LQG optimal control strategy also works to dynamically separate two sources [20].

2) Paper contribution: Here a three-source - fuel cell, battery and supercapacitor - power supply on board of an electric vehicle is considered. Each source is controlled by means of a DC-DC converter to operate in its frequency specialization range; the electrical motor with its converter is the load (Fig. 1.a). Fuel cell and battery are thus protected from strong variations of power demand. The EMS design is cast into the linear parameter varying (LPV) $/ \mathcal{H}_{\infty}$ framework methodology developed by authors in [21], which is a generic solution of EMS for multi-source systems with potentially any number of sources, that guarantees closed-loop robust stability. In this paper, the validation is done on a dedicated test-bench designed in collaboration with FEMTO-ST Laboratory in Belfort, France. Real battery and supercapacitor are used in the test bench, whereas a complete real-time emulator developed at FEMTO-ST Laboratory [22] represents the fuel cell. A reducedorder version of the controller [23] is employed in real time.

The methodology of implementation is discussed and comparison with the numerical simulation is conducted for IFSTTAR driving cycle (Institut Français des Sciences et Technologies des Transports, de l'Aménagement et des Réseaux), a suburban driving cycle. Effectiveness of the proposed EMS, as well as adaptations and limitations imposed by the real-world implementation, are discussed.

This paper is organized as follows. Section II provides a detailed description of the real-time test bench. Modelling of the LPV system, as well as control objectives, are presented in Section III. Section IV explains how the proposed LPV/H $\mathcal{H}_{\infty}$ control design procedure is adapted to the real-time test bench. Section V discusses the real-time validation results including a comparison with the numerical simulation ones. Section VI concludes the paper.

\section{DESCRIPTION OF THE REAL-TIME TEST BENCH}

The physical setup of the dedicated hardware-in-the-loop system corresponds to Fig. 1.b. The three power sources are paralleled on a DC-bus through DC-DC buck/boost converters; they supply an active load - whose variation is imposed to represent the desired driving cycle of the electric vehicle - by means of a rapidprototyping platform based on dSPACE MicroAutoBox ${ }^{\circledR}$ II device. Fig. 1.b shows an expanded view of the interconnections between the different parts used in the assembled test bench.

1) Power sources: This part is numbered as 1 in Fig. 1.b. The fuel cell is the main power source in the system. A 2-kW NEXA fuel cell with $50 \mathrm{~V}$ open-circuit voltage is here emulated by means of a fuel cell emulator [22]. In this way, behavior of the considered fuel cell can be replicated without hydrogen consumption. Consisting mainly of a programmable power supply driven by a real-time fuel cell model, the emulator is equipped by a human-machine interface, which allows on-line investigation of different parameters. Further details about fuel cell emulator can be found in [22], [24]. The supercapacitor is used to provide/absorb the fast variations of load current. Two MAXWELL supercapacitors in series are used to cover the voltage interval $[0,32] \mathrm{V}$, each of which has the capacity of $56 \mathrm{~F}$ with maximum voltage value of $16 \mathrm{~V}$. The supercapacitor voltage range is limited in between $[15,30] \mathrm{V}$, considered equivalent linearly to $[0,100] \%$ of state of charge (SOC). The battery is employed to handle the mid-range variations of the load current. Three batteries of $12 \mathrm{~V}$ and $110 \mathrm{Ah}$ are here connected in series.

2) $D C-D C$ converters: This part is numbered as 2 in Fig. 1.b. The fuel cell is connected to 1-quadrant DC-DC boost converter and the rest of sources are connected to 2-quadrant DCDC buck/boost converters to allow bi-directional power flows for charging/discharging. In this implementation, each of the three arms of a three-phase DC-AC inverter works as a different DCDC converter, each of which is connected to a common DC-bus. All inverter inputs/outputs can handle $25 \mathrm{~A} \mathrm{DC}$ current and up to $350 \mathrm{~V}$ DC voltage. Each DC-DC converter consists of two IGBT transistors operated by two complementary pulse-width modulated (PWM) signals. Averaged duty cycle of each PWM signal is noted by $\alpha$, where $\alpha \in[0,1]$. Practically, $\alpha \in[0.10,0.90]$ in order to avoid extreme operating conditions of the converters.

3) DC-bus: Numbered as 3 in Fig. 1.b, the DC-bus represents the physical connection between all paralleled power converters and the load. The DC-bus voltage should be regulated around a desired value regardless of load current variations. Here this voltage reference is chosen to be $V_{d c_{\text {ref }}}=60 \mathrm{~V}$, which can be ensured by all converters with duty cycle values in between admissible limits.

4) Active load: This part is numbered as 4 in Fig. 1.b; it is a device directly connected to the DC-bus, used to program a desired load current profile, a driving cycle profile in our case. The load current profile is provided as one-second-sampled table of values.

5) Sensors: This part is numbered as 5 in Fig. 1.b. Current sensors measure currents of all three sources $\left(I_{f c}, I_{b a t}\right.$ and $\left.I_{s c}\right)$ to be controlled at low level; at high-level control both DC-bus voltage $V_{d c}$ and supercapacitor voltage $V_{s c}$ are measured, where the last one is a direct image of supercapacitor SOC.

6) Real-time embedded control system: Numbered as 6 in Fig. 1.b, this part consists of the proposed EMS. To this end, MicroAutoBox ${ }^{\circledR}$ II from dSPACE is used as a flexible, MATLAB $^{\circledR} /$ Simulink $^{\circledR}$-programmable real-time system, effective for hardware-in-the-loop-based rapid prototyping. The sampling time, $50 \mu \mathrm{s}(20 \mathrm{KHz})$, is chosen in relation to the fastest dynamic in the system, namely that of PWM signals.

7) Human machine interface (HMI): This part is numbered as 7 in Fig. 1.b; it is designed using the ControlDesk 4.2 program and allows communication with the real-time control system. Its main tasks are to read and visualize the actual values of all interest variables. Some of parameters can also be tuned in real time.

\section{System MODELLING AND CONTROL OBJECTIVES}

\section{A. System modeling}

Fig. 1.a exhibits three main stages: the input stage including the power sources, the conversion stage including the DC-DC converters, and the DC link connected to the load as output stage. A global two-level control structure [21] is here used: all three sources are current-controlled within low-level proportional-integral (PI) controller-based loops whose references come from the upperlevel LPV/H $\mathcal{H}_{\infty}$-based controller (Fig. 2). Hence, low-level dynamics are much faster than the upper-level ones. Fuel cell and battery are modelled as ideal current sources, with negligible dynamics. 


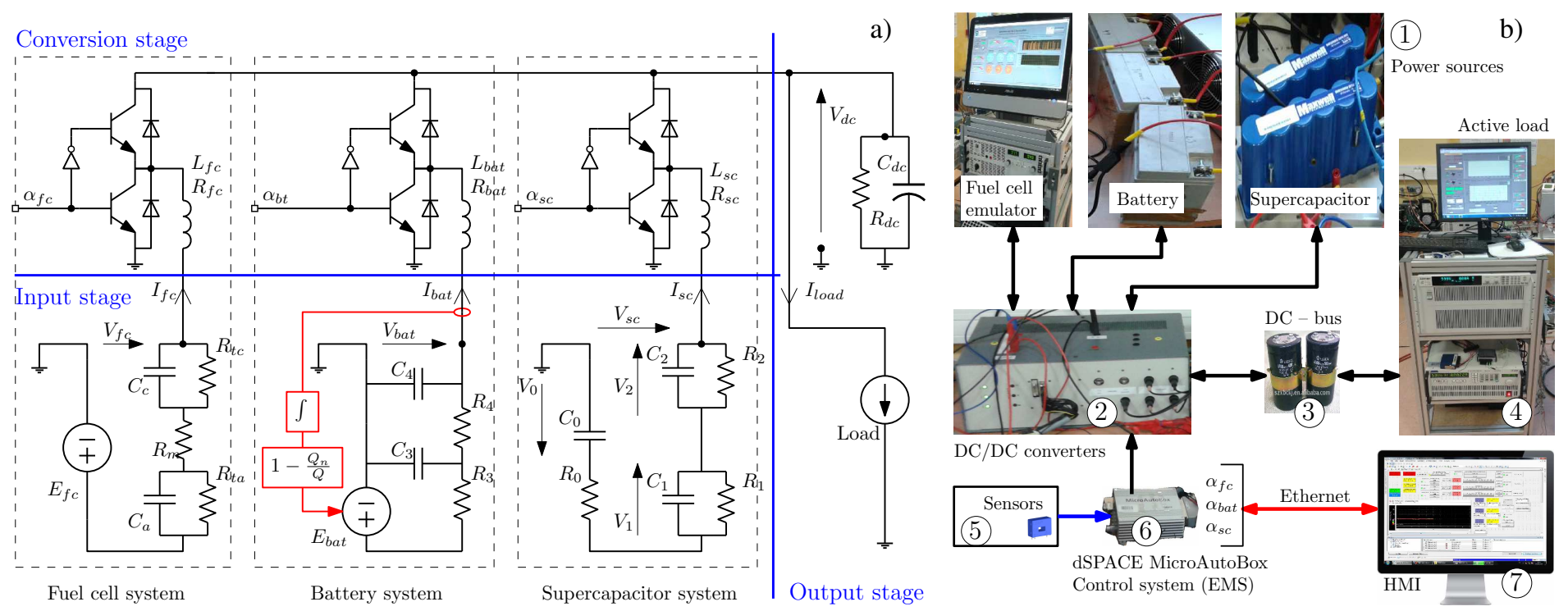

Figure 1: a) Electrical schematic of the considered on-board power supply system including power sources' equivalent electrical models. b) Schematics of the rapidprototyping test bench used to validate the energy management system.

Supercapacitor state of charge (SOC) is controlled to supply load variations during acceleration/braking phases. The EMS "sees" the load as the current drawn from or injected into the DC link. Average models of all the converters are used [25]. The global model is:

$$
\left\{\begin{aligned}
\frac{d I_{f c}}{d t}= & \frac{1}{L_{f c}}\left[V_{f c}-R_{f c} I_{f c}-V_{d c}\left(1-\alpha_{f c}\right)\right] \\
\frac{d I_{s c}}{d t}= & \frac{1}{L_{s c}}\left[V_{s c}-R_{s c} I_{s c}-V_{d c} \alpha_{s c}\right] \\
\frac{d I_{b a t}}{d t}= & \frac{1}{L_{b a t}}\left[V_{b a t}-R_{b a t} I_{b a t}-V_{d c} \alpha_{b a t}\right] \\
\frac{d V_{d c}}{d t}= & \frac{1}{C_{d c}}\left[\frac{-1}{R_{d c}} V_{d c}-I_{l o a d}+I_{f c}\left(1-\alpha_{f c}\right)\right. \\
& \left.+I_{b a t} \alpha_{b a t}+I_{s c} \alpha_{s c}\right],
\end{aligned}\right.
$$

where $I_{f c}, I_{b a t}$, and $I_{s c}$ are the currents of fuel cell, battery, and supercapacitor sources, respectively. $\alpha_{f c}, \alpha_{b a t}$, and $\alpha_{s c}$ are the corresponding converter average duty cycle values. $V_{f c}, V_{b a t}$ and $V_{s c}$ are fuel cell, battery and supercapacitor voltages, respectively. $L_{f c}, L_{b a t}, L_{s c}, R_{f c}, R_{b a t}$ and $R_{s c}$ are the inductances and resistances of smoothing inductors of each power converter, respectively. $I_{\text {Load }}$ is the load current, $V_{d c}$ is the DC-bus voltage, $C_{d c}$ and $R_{d c}$ are the DC-bus capacitor and resistance, respectively. $V_{s c}$ is the output of the supercapacitor electrical model in Fig. 1.a [26]:

$$
\left\{\begin{aligned}
\frac{d V_{0}}{d t} & =\frac{-1}{C_{0}} I_{s c} \\
\frac{d V_{1}}{d t} & =\frac{-1}{C_{1} R_{1}} V_{1}-\frac{1}{C_{1}} I_{s c} \\
\frac{d V_{2}}{d t} & =\frac{-1}{C_{2} R_{2}} V_{2}-\frac{1}{C_{2}} I_{s c} \\
V_{s c} & =-I_{s c} R_{0}+V_{0}+V_{1}+V_{2},
\end{aligned}\right.
$$

where $R_{0}, C_{0}, R_{1}, C_{1}, R_{2}$, and $C_{2}$ are constant parameters of supercapacitor model, $V_{0}, V_{1}$ and $V_{2}$ are sub-voltages in Fig. 1.a.

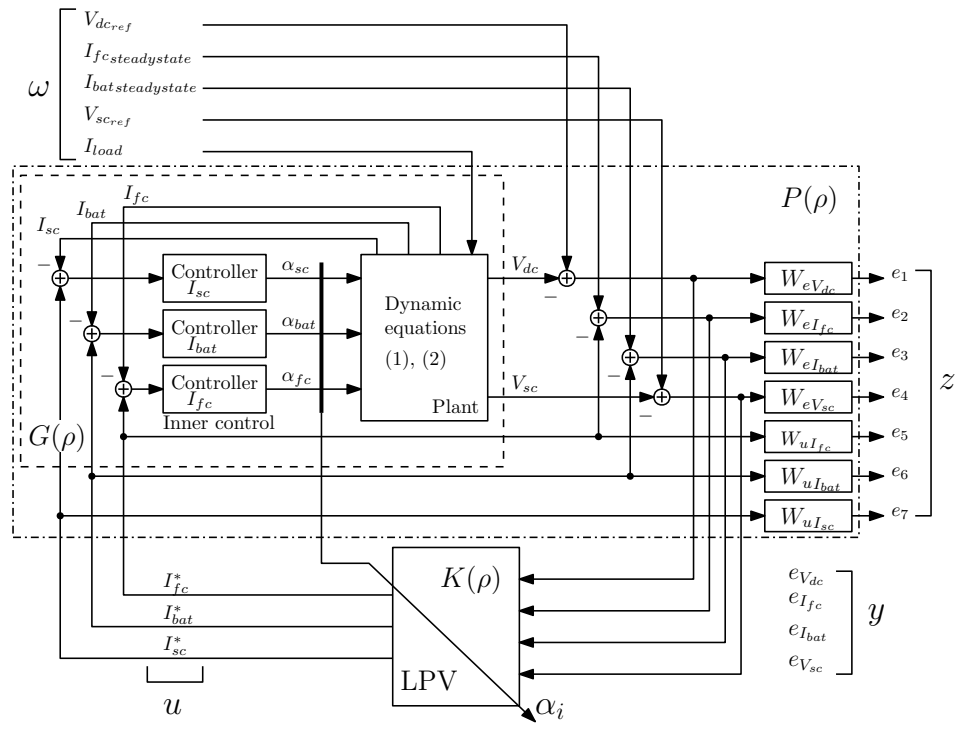

Figure 2: LPV/H $\mathcal{H}_{\infty}$ robust control design block diagram [21], [27].

The high-level control handles the dynamics in (2) and $V_{d c}$ dynamics from (1), while the three currents are controlled within the low-level control loops (PI current controllers - see Section IV-A). The system composed of (2) and $V_{d c}$ equation from (1) is rewritten in LPV form as follows:

$$
\left\{\begin{array}{llc}
\dot{x} & = & A \cdot x+B_{1} \cdot w+B_{2}(\rho) \cdot u \\
y & = & C \cdot x+D \cdot u
\end{array}\right.
$$

with state vector $x=\left[\begin{array}{llll}V_{d c} & V_{1} & V_{2} & V_{0}\end{array}\right]^{T}$, disturbance input $w=I_{\text {load }}$, control input vector $u=\left[\begin{array}{lll}I_{f c} & I_{b a t} & I_{s c}\end{array}\right]^{T}$ composed of fuel cell, battery and supercapacitor currents, respectively. $\rho=\left[\begin{array}{lll}\rho_{1} & \rho_{2} & \rho_{3}\end{array}\right]^{T}=\left[\begin{array}{lll}\alpha_{f c} & \alpha_{b a t} & \alpha_{s c}\end{array}\right]^{T}$ is the varying parameter vector, where each $\rho_{i}$ is bounded within $[0.1,0.9]$. Each varying parameter is supposed to be independent from the others. 


\section{B. Control objectives}

The main goals of the proposed EMS are:

1) to keep the DC-bus voltage around $60 \mathrm{~V}$ within an error of $\pm 10 \%$ regardless of the load current variations;

2) to apply dynamical (frequency) separation of power sources, i.e., each power source must supply power with respect to its characteristic frequency according to Ragone's classification [3] - this helps to protect fuel cell and battery from harmful fast variations of load current. Frequency separation is achieved due to some suitable choice of weighting functions associated to $\mathcal{H}_{\infty}$ control design (see Section IV-B);

3 ) to slowly keep the supercapacitor SOC around 50\%, allowing to absorb/provide power to fulfil instantaneous load power demand;

4) to impose a desired steady-state behavior for the fuel cell and battery, according to some desired power sharing between sources in steady state. Fuel cell operating point can thus be imposed, e.g., the maximum-efficiency one. Steady-state behavior is involved in battery charging, depending on its type. Battery charging could be achieved by using the main power source (fuel cell).

Note that battery SOC is not controlled; instead, its charging/discharging current regimes are imposed. Indeed, in practice a battery SOC is not controlled linearly around a desired value like supercapacitor's one, but it is used as an indicator to start its charging/discharging according to some desired regime (e.g., $\mathrm{C} / 5$ or $\mathrm{C} / 10$, where $\mathrm{C}$ is the battery capacity in $\mathrm{Ah}$ ).

\section{CONTROL DESIGN: SELECTION OF PARAMETERS AND ADAPTATIONS TO THE TEST BENCH}

\section{A. Curent control loops design (low-level control)}

Each source current must be controlled and prevented from exceeding admissible limits. Supposing that the DC-link voltage $V_{d c}$ is kept constant at its reference value $V_{d c_{r e f}}$ by the EMS, dynamics of currents (1) are linear, which justifies their control by classical PI controllers. These loops are designed to be fast enough to be neglected at the slower-dynamic upper level and to ensure tracking of current references generated by upper-level LPV $/ \mathcal{H}_{\infty}$ controller (Fig. 2). Hence, the following equalities hold in the sequel: $I_{f c}{ }^{*}=I_{f c}, I_{b a t}{ }^{*}=I_{b a t}$ and $I_{s c}{ }^{*}=I_{s c}$. PI controllers' parameters can be found in Table II in the Appendix.

The three tracking low-level loops have the converters' duty cycles, $\alpha_{f c}, \alpha_{b a t}, \alpha_{s c}$, as control inputs. It is thus reasonable to choose the varying parameter vector $\rho=\left[\begin{array}{lll}\alpha_{f c} & \alpha_{b a t} & \alpha_{s c}\end{array}\right]^{T}$ to describe globally the time-varying system.

\section{B. $L P V / \mathcal{H}_{\infty}$ control loop design (upper-level control)}

The LPV controller $K(\rho)$ in Fig. 2 should satisfy the control objectives in Section III-B for the parameter-varying plant $G(\rho)$; its design is tackled in the $\mathcal{H}_{\infty}$ framework applied to LPV systems [28]. In Fig. 2 usual forms of the weighting functions are considered to represent the control objectives. A first-order weighting function $1 / W_{e V_{d c}}$ is in charge to ensure performance of load variations rejection, i.e., both desired time response and acceptable error range for the DC-link voltage $\left(V_{d c}\right)$ regulation. A first-order weighting function $1 / W_{e V_{s c}}$ is used to maintain supercapacitor SOC around $50 \%$. A supercapacitor's SOC may be expressed as:

$$
S O C=\left(V_{s c}-V_{s c, \min }\right) /\left(V_{s c, \max }-V_{s c, \min }\right) \cdot 100 \%,
$$

where $V_{s c, \max }$ is the maximum allowed supercapacitor's voltage (SOC $=100 \%$ ) and $V_{s c, \text { min }}$ is the minimum allowed voltage input for the supercapacitor converter $(\mathrm{SOC}=0 \%)$. Here $V_{s c} \in[15,30] \mathrm{V}$.

Weighting functions $1 / W_{u I f c}, 1 / W_{u I b a t}$ and $1 / W_{u I s c}$ shape the dynamic behavior of current references of fuel cell, battery and supercapacitor, respectively, in order to dynamically separate the sources depending on their characteristic frequencies. Sharp separation is done with fourth-order functions $1 / W_{u \text { Ibat }}$ and $1 / W_{u I s c}$, while $1 / W_{u I f c}$ is a constant. Weighting function $1 / W_{e_{I_{f c}}}$ is defined as a first-order one and $1 / W_{e I_{b a t}}$ is a constant value to determine fuel cell and battery long-term behaviors, respectively. Thus, a desired steady-state power sharing can be imposed by using $I_{f c_{\text {steadystate }}}$ and $I_{\text {bat steadystate }}$ reference inputs. Plant $G(\rho)$ together with the weighting functions form the extended plant $P(\rho)$.

Parameters of weighting functions result from a geneticalgorithm (GA)-based optimization procedure [29], [30] with a twofold objective function: to guarantee an arbitrarily imposed degree of closed-loop stability, while sizing a desired frequency separation between power sources according to each application [27]. Guess values for this algorithm are selected as presented in Section IV-D3. The resulting weighting functions are given in the Appendix.

\section{C. $L P V / \mathcal{H}_{\infty}$ controller design and order reduction}

The control design problem is cast into a set of Linear Matrix Inequalities (LMI) and solved in the context of LPV/H $\mathcal{H}_{\infty}$ control polytopic approach [31]. System in (3) has a polytopic form with $2^{3}=8$ vertices (since the parameter vector $\rho$ has three bounded elements in $[0.1,0.9])$. The generalized LPV MIMO system is:

$$
\left[\begin{array}{l}
\dot{x} \\
z \\
y
\end{array}\right]=\left[\begin{array}{ccc}
A & B_{1} & B_{2}(\rho) \\
C_{1} & D_{11} & D_{12} \\
C_{2} & D_{21} & D_{22}
\end{array}\right]\left[\begin{array}{l}
x \\
w \\
u
\end{array}\right] .
$$

The LMIs are solved off-line by using Yalmip/Sedumi solver as a convex optimization with a single Lyapunov function (framework of quadratic stabilization [28], [32]) at each vertex of the polytope, leading to vertex controllers $K_{i}=\left[\begin{array}{ll}A_{i} & B_{i} \\ C_{i} & D_{i}\end{array}\right]$ with $1 \leq i \leq 8$. The LPV controller $K(\rho)$ is computed on-line as a convex combination of the vertex controllers $K_{i}$, i.e., $K(\rho)=\sum_{1}^{8} \gamma_{i}(\rho) K_{i}$, with:

$$
\gamma_{i}(\rho)=\frac{\prod_{j=1}^{3}\left|\rho_{j}-C\left(w_{i}\right)_{j}\right|}{\prod_{j=1}^{3}\left|\overline{\rho_{j}}-\underline{\rho_{j}}\right|}>0, \quad \sum_{1}^{8} \gamma_{i}=1
$$

where $w_{i}$ are the vertices corresponding to the extreme values of the parameter vector $\rho . C\left(w_{i}\right)_{j}$ is the $j^{t h}$ component of the vector $C\left(w_{i}\right)$ defined as $C\left(w_{i}\right)_{j}= \begin{cases}\overline{\rho_{j}} & \text { if } w_{i}=\rho_{j} \\ \underline{\rho_{j}} & \text { otherwise }\end{cases}$ $\overline{\rho_{j}}=\max \left(\rho_{j}\right)=0.9, \rho_{j}=\min \left(\rho_{j}\right)=0.1$.

The full-order $\mathrm{LPV} / \mathcal{H}_{\infty}$ controller is a combination of eight eighteenth-order controllers. For practical purposes, MORE toolbox [33] is used to find a reduced-order model that fits the original controller for bounded frequency range. The Iterative SVD Tangential Krylov Algorithm (ISTIA) is applied on each vertex controller for the whole closed-loop bandwidth, resulting in tenth-order vertex controllers. Stability and performance have been checked a posteriori (see [23] for more details). 


\section{Adaptations to the test bench}

1) Control design: The proposed methodology has been applied to the test bench model to obtain a reduced-order controller for real-time implementation. Sources' currents saturation have been taken into account in the design. Comparison between real-time and numerical simulation results has been performed under the same scenario. Thus, samples of the measured load current (Fig. 3.a) were used to re-perform numerical simulation.

2) Selection of control references: Four exogenous inputs represent the references for the upper-level control loop (Fig. 2). These references are selected for real-time implementation as follows:

a) DC-bus voltage reference $V_{d c_{r e f}}$ is chosen to be $60 \mathrm{~V}$;

b) fuel cell steady-state behavior is imposed such that fuel cell be able to supply the total average load current, according to:

$$
I_{f c, r e f}(t)=\operatorname{average}\left(I_{\text {load }}(t)\right) /\left(1-\alpha_{f c}(t)\right),
$$

where average $\left(I_{\text {load }}(t)\right)$ is the load current suitably low-pass filtered, e.g., with $10 \mathrm{rad} / \mathrm{s}$ as cut-off frequency to remove noise. $\alpha_{f c}(t)$ is the real-time value of the fuel cell converter average duty cycle. However, this reference choice is not unique and the fuel cell can be managed in a different way, e.g., to provide quasi-constant current corresponding to its maximum power efficiency;

c) as the fuel cell provides all the load power in steady state, then the battery is here managed to provide no current in long term, thus preserving its SOC: $I_{b a t_{r e f}}=0 \mathrm{~A}$. However, the battery should be able to replace the fuel cell on line in case of unavailability - in this case, another proper choice of $I_{b a t_{r e f}}$ must be made;

d) supercapacitor SOC reference is reasonably chosen as $50 \%$, so the supercapacitor is always ready to either provide or absorb power in case of fast and important variations of load current.

3) Selection of weighting functions: This step is crucial for implementing a reliability-aware design of the proposed EMS. Selection of $1 / W_{e V_{d c}}, 1 / W_{e V_{s c}}, 1 / W_{e I_{f c}}$ and $1 / W_{e I_{b a t}}$ is explained above in Section IV-B. As regards the weighting functions $1 / W_{u I_{f c}}, 1 / W_{u I_{b a t}}$ and $1 / W_{u I_{s c}}$, they must be chosen to represent a suitable conditioning of the source currents' response to load variations. Standard forms are here chosen, whose parameters - mainly their folding frequencies - result from a GA-based optimization, as explained above. Initial guess values are selected as follows.

Basically, the folding frequencies of these filters are chosen in relation with the characteristic frequencies of sources, which quantitatively express the maximum speed of variations each source can provide and still respect its reliability specifications. Practically, for a given storage technology, its characteristic frequency may be deduced from the datasheet, i.e., from the maximum current gradient recommended for maximizing its proper operation time.

Therefore, the fuel cell current must only have slow variations, so $1 / W_{u I_{f c}}$ will be a low-pass filter with a roll-off frequency near its characteristic frequency. Battery current variations will be confined within a bandwidth including its characteristic frequency, so $1 / W_{u I_{b a t}}$ will be a bandpass filter. Finally, the ultracapacitor must work at high-frequency variations, so $1 / W_{u I_{s c}}$ will ideally be a high-pass filter with a folding frequency near its characteristic frequency. In practice, however, it is about a bandpass filter, given the limited spectrum of supercapacitor. $1 / W_{u I_{f c}}, 1 / W_{u I_{b a t}}$ and $1 / W_{u I_{s c}}$ may be locally superposed, more or less - in frequency regions where the load is supplied by two of the three sources - and together they should cover the whole spectrum of load variations.

4) Expected differences between numerical simulation and realtime results: The modelling uncertainty of sources is expected to be the main source of differences here, that is, differences between nominal and real values of parameters characterizing each of the three sources. Indeed, widely-known equivalent electrical models of literature have been used in simulation for the fuel cell, battery and supercapacitor, with datasheet values of parameters of these models. It is however known that such parameters - resistances, inductances, capacities - vary in time and may not be equal to the datasheet ones any longer. Resuming identification of sources' models was beyond the scope of this paper. Therefore, currents of sources are expected to vary in real time differently from in simulation. Meanwhile, the proposed control strategy is expected to perform robustly against these parameter variations.

\section{REAL-TIME VALIDATION RESULTS}

This section discusses the real-time results obtained on the test bench. Different driving cycles can be used for EMS assessment. Here the active load implements the extra-urban IFSTTAR driving cycle, where acceleration, speed variation on high-way, deceleration and full brake are frequent (Fig. 3.a); its rich frequency content is illustrative for the frequency separation of sources (see Section $\mathrm{V}-2$ ). Results are analyzed in both time and frequency domains.
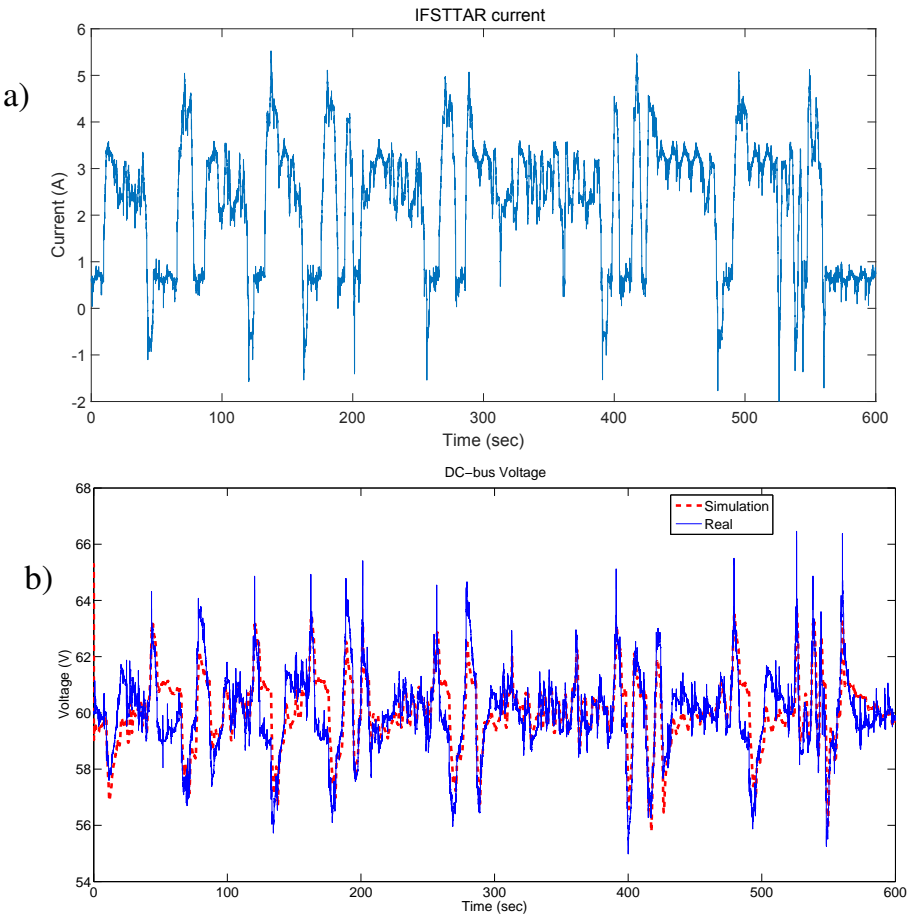

Figure 3: a) Load current scenarii representing the IFSTTAR driving cycle, as provided by the programmable power supply/load used in the test bench. b) Realtime versus numerical-simulation behavior of the regulated DC-bus voltage in response to IFSTTAR driving cycle.

1) Time-domain analysis: As mentioned before, the measured load current $I_{\text {load }}$ (Fig. 3.a) is low-pass filtered and used in numerical simulation. Real-time results are here compared against 
a)

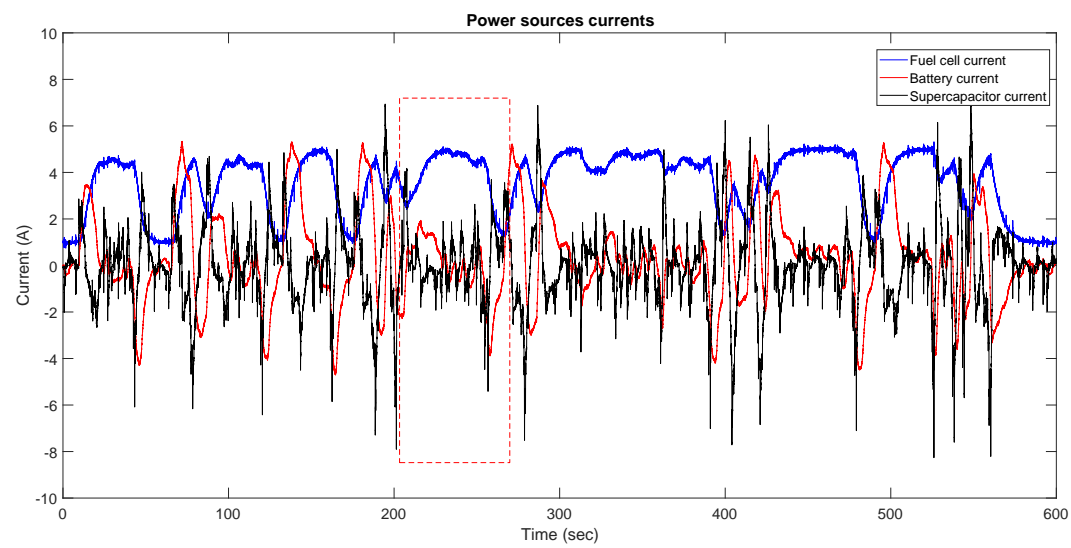

b)

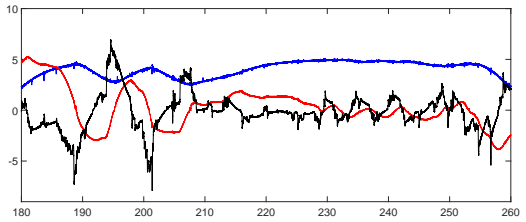

Figure 4: a) The three real-time power sources' currents corresponding to IFSTTAR driving cycle. b) Zoom.

a)

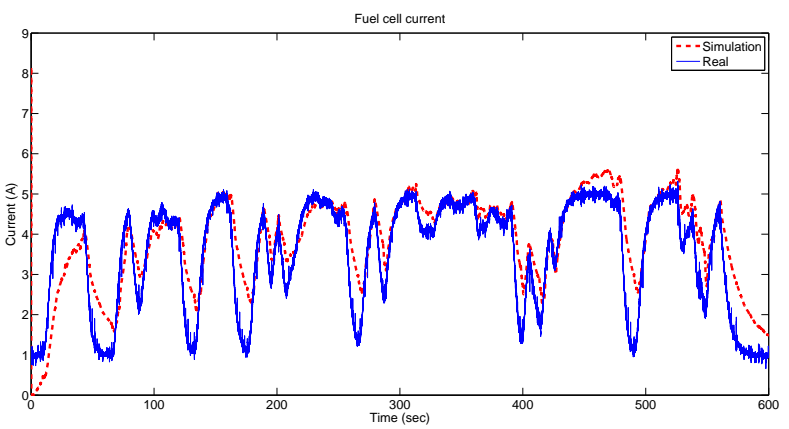

b)

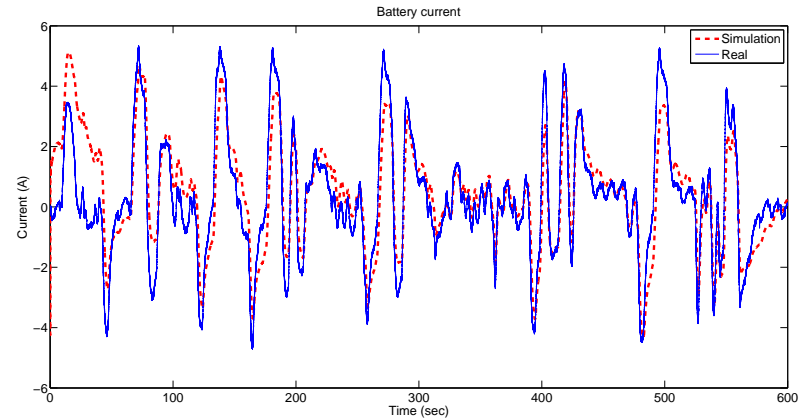

Figure 5: Real-time versus numerical-simulation behavior of a) fuel cell current and b) battery current in response to IFSTTAR driving cycle.

simulation ones; Table I quantifies these differences by means of normalized difference signal Euclidean norms of each variable of interest: $V_{d c}, I_{f c}, I_{b a t}, I_{s c}, S O C_{s c}$. Time-domain analysis is performed in relation to the control objectives in Section III-B.

Table I: Quantification of differences between simulation and real-time results, based on normalized difference signal norms: $\operatorname{dif} f_{x}=\frac{\left\|x_{\text {realtime }}-x_{\text {simulation }}\right\|_{2}}{\left\|x_{\text {simulation }}\right\|_{2}}$.

\begin{tabular}{|c|c|c|c|c|c|}
\hline & $V_{d c}$ & $I_{f c}$ & $I_{b a t}$ & $I_{s c}$ & $S O C_{s c}$ \\
\hline $\operatorname{diff}_{x}[\%]$ & 2.3 & 22.37 & 22.34 & 19.19 & 6.7 \\
\hline
\end{tabular}

DC-link voltage control (first control objective): Fig. 3.b shows the $V_{d c}$ time evolution. Note that real-time results differ by just $2.3 \%$ from simulation, due to filtering for noise reduction, and show $V_{d c}$ regulation within the imposed boundaries $(60 \mathrm{~V} \pm 10 \%)$.

Dynamical separation of the power sources (second control ob- a)

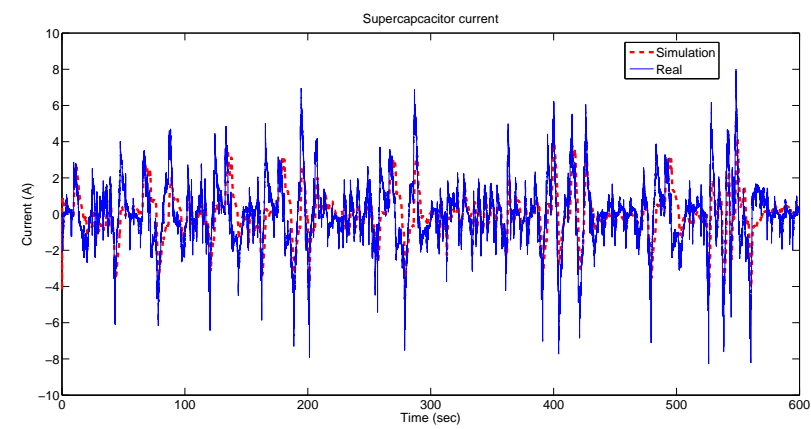

b)

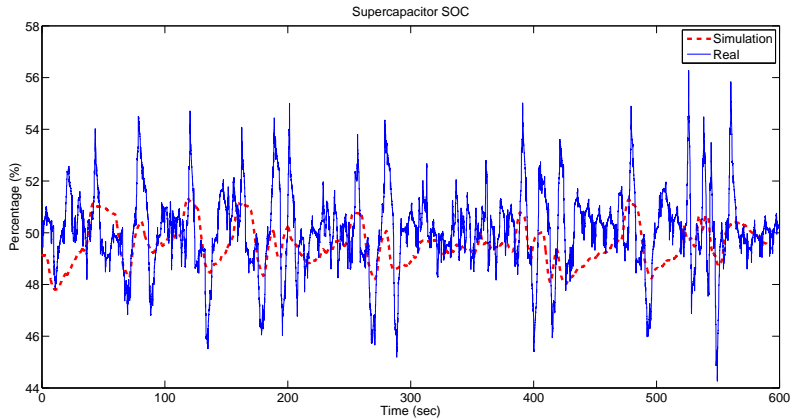

Figure 6: Real-time versus numerical-simulation behavior of a) supercapacitor current and b) supercapacitor SOC corresponding to IFSTTAR driving cycle.

jective): evolutions of the three currents in Fig. 4 show that fuel cell, battery and supercapacitor operate in low-, mid- and high-frequency range, respectively. As expected, fuel cell current is smoother compared to battery and supercapacitor ones. Supercapacitor current has the fastest variation, which complies with its role of high-frequency (high-power-density) source. Frequency-domain analysis in Section V-2 illustrates better the frequency separation goal. Fig. 5.a shows the time evolution of fuel cell current. Real-time results differ from simulation by around 20\%; note also that their initial states are different. This can also be noticed for the battery current evolution shown in Fig. 5.b. Supercapacitor provided/absorbed current is shown in Fig. 6.a. Here, the difference between real time and simulation, especially concerning variations' magnitude, are just under $20 \%$. As predicted, the largest differences between real time and simulation can effectively be seen in the behavior of sources' 
currents. This is potentially due to imprecise models and/or to differences between datasheet and real parameter values, as well as to noise from different sources (sensors, converters, etc.).

Supercapacitor SOC regulation (third control objective): Fig. 6.b shows that, as expected, the supercapacitor SOC is maintained around $50 \%$. Table I shows that differences between the SOC realtime behavior and simulation are less than $10 \%$; they are due to use of (4) in SOC computation: supercapacitor voltage is smoother in numerical simulation as average converter models are used. Despite these differences, the SOC is well regulated in both numerical simulation and real time, varying at maximum $\pm 6 \%$ around the desired reference (50\%). Hence, the system is able to handle larger load current variations; otherwise, a smaller supercapacitor can be used instead. Varying parameter vector $\rho=\left[\alpha_{f c}, \alpha_{b a t}, \alpha_{s c}\right]^{T}$ exhibits significant variations, but without reaching saturation.

2) Frequency-domain analysis: To illustrate the frequency separation feature of the proposed EMS, the power spectral density (PSD) and its normalized version are calculated for all current signals, including the load current $\left(I_{f c}, I_{b a t}, I_{s c}, I_{l o a d}\right)$ :

$$
\left\{\begin{array}{l}
P S D(x)=(1 / N) \sqrt{\tilde{X}(f) \tilde{X}(f)^{*}} \\
\text { NormalizedPSD }=(P S D / \max (P S D)) \cdot 100 \%
\end{array}\right.
$$

where $\tilde{X}(f)$ is the discrete Fourier transform of the discrete-time signal $x(k)$ of length $N . \tilde{X}(f)^{*}$ is the complex-conjugated of $\tilde{X}(f)$. The normalized PSD of a source allows to identify the frequency range where most of that source's power is placed. Fig. 7 shows the normalized PSD of all three currents $\left(I_{f c}, I_{b a t}, I_{s c}\right)$ for IFSTTAR driving cycle. Fuel cell maximum power is provided at very low frequencies within $\left[0, \omega_{1}\right]=[0,0.1] \mathrm{rad} / \mathrm{s}$, practically representing a DC value. The battery maximum power is delivered within the range next to the fuel cell's one, i.e., $\left[\omega_{4}, \omega_{5}\right]=[0.1,0.25] \mathrm{rad} / \mathrm{s}$. Finally, as expected, the supercapacitor maximum use is within $[0.25,0.6] \mathrm{rad} / \mathrm{s}$, i.e., in high frequency compared to the other two sources. Supercapacitor maximum power must usually be provided depending on the maximum frequency in the load spectrum.

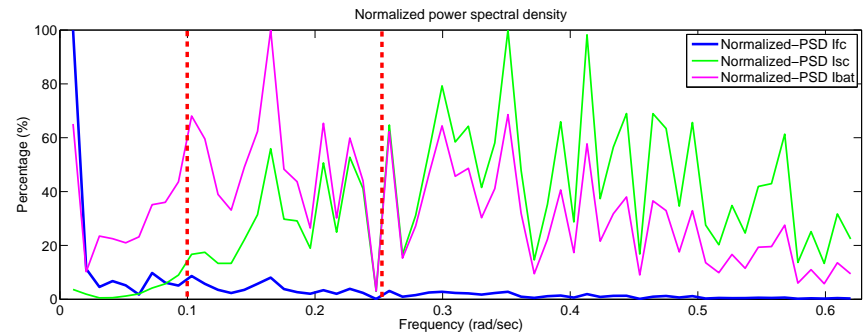

Figure 7: Normalized power spectral density (PSD) of the three sources' currents under the IFSTTAR driving cycle scenario.

\section{CONCLUSION}

In this paper, the real-time validation of a multi-variable energy management system (EMS) based on LPV/H $\mathcal{H}_{\infty}$ control is presented. A reduced-order version of a systematically designed $\mathrm{LPV} / \mathcal{H}_{\infty}$ controller has been implemented on a dedicated test bench representing a three-source - fuel cell, battery and supercapacitor power supply system on board of an electric vehicle. Real battery and supercapacitor have been used, while the fuel cell has been represented by a complete emulator. Sources are paralleled on a DC link by means of DC-DC converters; the DC bus feeds the load represented by the vehicle's electrical motor. A dSPACE MicroAutoBox $^{\circledR}$ II device embeds the designed EMS, due to its flexibility and ease of programming with MATLAB ${ }^{\circledR}$.

The EMS performance has been assessed against the imposed control objectives - DC-bus voltage regulation, dynamical separation of sources' current variations depending on specialization of each source, and imposing desired steady-state behavior for each source - with very promising results. IFSTTAR (Institut Français des Sciences et Technologies des Transports, de l'Aménagement et des Réseaux) driving cycle has been here chosen for illustration, due to its rich frequency content able to challenge all the three sources and thus to allow an as meaningful as possible validation. The EMS design does not require any previous knowledge of the load current, so the proposed strategy is not driving-cycle dependent.

Further work may aim at integrating some preliminary Fourier analysis results about the load profile, e.g., to find the maximum frequency in its spectrum. Further studies may also exploit the quasidiagonal form of the reduced-order $\mathrm{LPV} / \mathcal{H}_{\infty}$ controller transfer matrix, consistent with the almost independent behavior of sources when paralleled on the DC link. A structured $\mathcal{H}_{\infty}$ control design may be interesting in this case, where a certain desired controller structure can a priori be imposed and optimized.

\section{ACKNOWLEDGMENT}

The authors would like to thank Professor Fei GAO, Mr. Patrice LUBERDA, and Mr. Pierre DIEBOLT for their help to apply the proposed methodology to the considered real-time application.

\section{APPENDIX}

$$
\left\{\begin{aligned}
\frac{1}{W_{e V_{d c}}} & =\frac{s+\omega_{b} \cdot \epsilon}{s / M_{s}+\omega_{b}}=\frac{s+0.05}{0.5363 s+500} \\
\frac{1}{W_{e I_{f c}}} & =\frac{s+\omega_{B C} \cdot \epsilon}{s / M_{u}+\omega_{B C}}=\frac{s+0.0007}{0.9091 s+0.07} \\
\frac{1}{W_{e I_{b a t}}} & =1.9 \\
\frac{1}{W_{e V_{s c}}} & =\frac{s+\omega_{b} \cdot \epsilon}{s / M_{s}+\omega_{b}}=\frac{s+0.0005}{0.5263 s+0.05} \\
\frac{1}{W_{u I_{f c}}} & =3 \\
\frac{1}{W_{u I_{b a t}}} & =\left(\frac{\epsilon \cdot s+\omega_{B C}}{s+\omega_{B C} / M} \cdot \frac{s+\omega_{b} \cdot \epsilon}{s / M+\omega_{b}}\right)^{2} \\
& =\frac{s^{4}+57.14 s^{3}+816.3 s^{2}+0.005714 s+10^{-8}}{0.34 s^{4}+0.0172 s^{3}+2.858 \cdot 10^{-4} s^{2}+1.721 \cdot 10^{-6} s+3.4 \cdot 10^{-9}} \\
\frac{1}{W_{u I_{s c}}} & =\left(\frac{\epsilon \cdot s+\omega_{B C}}{s+\omega_{B C} / M} \cdot \frac{s+\omega_{b} \cdot \epsilon}{s / M+\omega_{b}}\right)^{2} \\
& =\frac{s^{4}+395 s^{3}+3.907 \cdot 10^{4} s^{2}+1.185 \cdot 10^{4} s+900}{0.1413 s^{4}+19.91 s^{3}+709.5 s^{2}+597.2 s+127.2}
\end{aligned}\right.
$$

\section{REFERENCES}

[1] J. Guerrero, M. Chandorkar, V. Lee, and P. Loh, "Advanced control architectures for intelligent microgrids - part i: Decentralized and hierarchical control," IEEE Transactions on Industrial Electronics, vol. 60(4), pp. 1254-1262, 2013. 
Table II: Electrical system parameters used for simulation and real-time validation (notations refer to Fig. 1.a).

\begin{tabular}{|c|c|c|}
\hline Parameter & Description & Real-time \\
\hline DC-bus & & validation value \\
\hline$V_{d c}$ & DC-bus voltage & $60 \mathrm{~V}$ \\
\hline$C_{d c}$ & DC-bus capacitor & $6.6 \cdot 10^{-3} F$ \\
\hline$R_{d c}$ & DC-bus discharging resistor & $10.0 \cdot 10^{+3} \Omega$ \\
\hline \multicolumn{3}{|l|}{ Fuel cell } \\
\hline$L_{f c}$ & Fuel cell converter's inductor & $2.0 \cdot 10^{-3} H$ \\
\hline$R_{f c}$ & Fuel cell converter's resistor & $1.0 \cdot 10^{-2} \Omega$ \\
\hline$E_{f c}$ & Fuel cell open-circuit voltage & $50 \mathrm{~V}$ \\
\hline$R_{m}$ & Fuel cell model's resistor & $7.63 \cdot 10^{-2} \Omega$ \\
\hline$R_{t a}$ & Fuel cell model's resistor & $2.0 \cdot 10^{-3} \Omega$ \\
\hline$R_{t c}$ & Fuel cell model's resistor & $4.72 \cdot 10^{-4} \Omega$ \\
\hline$C_{a}$ & Fuel cell model's capacitor & $2.12 \cdot 10^{-3} \mathrm{~F}$ \\
\hline$C_{c}$ & Fuel cell model's capacitor & $2.12 \cdot 10^{-2} F$ \\
\hline \multicolumn{3}{|l|}{ Battery } \\
\hline$L_{\text {bat }}$ & Battery converter's inductor & $1.0 \cdot 10^{-3} H$ \\
\hline$R_{\text {bat }}$ & Battery converter's resistor & $2.0 \cdot 10^{-3} \Omega$ \\
\hline$Q_{n}$ & Initial SOC of battery & $0 \%$ \\
\hline$C_{3}$ & Battery model's capacitor & $2.92 \cdot 10^{-4} F$ \\
\hline$R_{3}$ & Battery model's resistor & $13.4 \cdot 10^{-3} \Omega$ \\
\hline$C_{4}$ & Battery model's capacitor & $2.92 \cdot 10^{-4} \mathrm{~F}$ \\
\hline$R_{4}$ & Battery model's resistor & $13.4 \cdot 10^{-3} \Omega$ \\
\hline \multicolumn{3}{|l|}{ Supercapacitor } \\
\hline$L_{s c}$ & Supercapacitor converter's inductor & $1.0 \cdot 10^{-3} H$ \\
\hline$R_{s c}$ & Supercapacitor model's resistor & $0.8 \cdot 10^{-3} \Omega$ \\
\hline$C_{0}$ & Supercapacitor model's capacitor & $56.0 F$ \\
\hline$C_{1}$ & Supercapacitor model's capacitor & $1.0 F$ \\
\hline$C_{2}$ & Supercapacitor model's capacitor & $1.0 F$ \\
\hline$R_{1}$ & Supercapacitor model's resistor & $6.0 \cdot 10^{-4} \Omega$ \\
\hline$R_{2}$ & Supercapacitor model's resistor & $4.5 \cdot 10^{-4} \Omega$ \\
\hline \multicolumn{3}{|l|}{ PIs local control loops } \\
\hline$K p_{f c}, K i_{f c}$ & Fuel cell current control loop & $0.5,0.1$ \\
\hline$K p_{\text {bat }} \quad, \quad K i_{\text {bat }}$ & Battery current control loop & $0.01,0.8$ \\
\hline$K p_{s c} \quad, \quad K i_{s c}$ & Supercapacitor current control loop & $0.01,0.4$ \\
\hline
\end{tabular}

[2] J. Justo, F. Mwasilu, J. Lee, and J. W. Jung, "Ac-microgrids versus dcmicrogrids with distributed energy resources: A review," Renewable and Sustainable Energy Reviews, vol. 24, pp. 387-405, 2013.

[3] A. Kuperman and I. Aharon, "Battery/ultracapacitor hybrids for pulsed current loads: A review,” Renewable and Sustainable Energy Reviews, vol. 15, pp. 981992, Feb. 2011.

[4] T. Azib, C. Larouci, A. Chaibet, and M. Boukhnifer, "Online energy management strategy of a hybrid fuel cell/battery/ultracapacitor vehicular power system," IEEJ Transactions on Electrical and Electronic Engineering, vol. 9, pp. 548-554, Sept. 2014.

[5] S. F. Tie and C. W. Tan, "A review of energy sources and energy management system in electric vehicles," Renewable and Sustainable Energy Reviews, vol. 20, pp. 82-102, Apr. 2013.

[6] I. Aharon and A. Kuperman, "Topological overview of powertrains for batterypowered vehicles with range extenders," IEEE Transactions on Power Electronics, vol. 26, pp. 868-876, Mar. 2011.

[7] D. Iannuzzi, "Use of Supercapacitors, Fuel cells and Electrochemical Batteries for Electric Road Vehicles: A Control Strategy," in 33rd Annual Conference of the IEEE Industrial Electronics Society, 2007. IECON 2007, pp. 539-544, Nov. 2007.

[8] S. Schuler, D. Schlipf, P. W. Cheng, and F. Allgöwer, "11-optimal control of large wind turbines," IEEE Transactions on Control Systems Technology, vol. 21(4), pp. 1079-1089, 2013.

[9] A. M. Dizqah, A. Maheri, K. Busawon, and P. Fritzson, "Standalone de microgrids as complementarity dynamical systems: Modeling and applications," Control Engineering Practice, vol. 35, pp. 102-112, 2015.

[10] S. Xie, H. He, and J. Peng, "An energy management strategy based on stochastic model predictive control for plug-in hybrid electric buses," Applied Energy, vol. 196, pp. 279-288, 2017.

[11] M. Rajabzadeh, S. M. T. Bathaee, and M. A. Golkarz, "Dynamic modeling and nonlinear control of fuel cell vehicles with different hybrid power sources," International Journal of Hydrogen, vol. 41, pp. 3185-3198, 2016.

[12] T. Alnejaili, S. Drid, D. Mehdi, L. Chrifi-Alaoui, and H. Sahraoui, "Sliding mode control of a multi-source renewable power system," in Proceedings of
2015 3rd International Conference on Control, Engineering \& Information Technology - CEIT 2015, Tiemcen, Algeria, May 25-27 2015, 2015.

[13] M. R. B. Khan, R. Jidin, and J. Pasupuleti, "Multi-agent based distributed control architecture for microgrid energy management and optimization," Energy Conversion and Management, vol. 112, pp. 288-307, 2016.

[14] F. Martel, S. Kelouwani, Y. Dubé, and K. Agbossou, "Optimal economy-based battery degradation management dynamics for fuel-cell plug-in hybrid electric vehicles," Journal of Power Sources, vol. 274, pp. 367-381, 2015.

[15] X. Xu, H. Jia, D. Wang, D. C. Yu, and H.-D. Chiang, "Hierarchical energy management system for multi-source multi-product microgrids," Renewable Energy, vol. 78, pp. 621-630, 2015.

[16] A. Castaings, W. Lhomme, R. Trigui, and A. Bouscayrol, "Decomposed energy management of a multi-source fuel cell vehicle using energetic macroscopic representation," in Proceedings of 2016 IEEE Vehicle Power and Propulsion Conference - VPPC 2016, Hangzhou, China, Oct. 17-20 2016, 2016.

[17] M. Silva, J. Trovao, P. Pereirinha, and H. Jorge, "Application of a decoupling method based on online filtering technique for multi-source electric vehicles," in 2013 15th European Conference on Power Electronics and Applications (EPE), pp. 1-10, Sept. 2013.

[18] A. Florescu, S. Bacha, I. Munteanu, and A. I. Bratcu, "Frequency-separationbased energy management control strategy of power flows within electric vehicles using ultracapacitors," in IECON 2012 - 38th Annual Conference on IEEE Industrial Electronics Society, pp. 2957-2964, Oct. 2012.

[19] A. Florescu, S. Bacha, I. Munteanu, A. I. Bratcu, and A. Rumeau, "Adaptive frequency-separation-based energy management system for electric vehicles," Journal of Power Sources, vol. 280, pp. 410-421, Apr. 2015.

[20] A. Florescu, A. I. Bratcu, I. Munteanu, A. Rumeau, and S. Bacha, "LQG Optimal Control Applied to On-Board Energy Management System of All-Electric Vehicles," IEEE Transactions on Control Systems Technology, vol. 23(4), no. 99, pp. 1427-1439, 2015.

[21] W. Nwesaty, A. I. Bratcu, and O. Sename, "Power sources coordination through multivariable linear parameter-varying/ control with application to multi-source electric vehicles," IET Control Theory and Applications, vol. 10(16), pp. 20492059, 2016.

[22] F. Gao, B. Blunier, M. G. Simões, and A. Miraoui, "PEM fuel cell stack modeling for real-time emulation in hardware-in-the-loop applications," IEEE Transactions on Energy Conversion, vol. 26, no. 1, pp. 184-194, 2011.

[23] W. Nwesaty, A. I. Bratcu, and O. Sename, "Reduced-order LPV controller for coordination of power sources within multi-source energy systems," in Proceedings of 8th IFAC Symposium on Robust Control Design (ROCOND'15), pp. 131-136, 2015.

[24] G. Fei, B. Benjamin, and M. Abdellatif, Proton Exchange Membrane Fuel Cells Modeling. Wiley-ISTE, 2012.

[25] S. Bacha, I. Munteanu, and A.I. Bratcu, Power Electronics Converters Modelling and Control: With Case Studies. London: Springer, "Advanced $T$ extbooks in Control and Signal Processing Series" ed., 2013.

[26] C. H. Wu, Y. H. Hung, and C. W. Hong, "On-line supercapacitor dynamic models for energy conversion and management," Energy Conversion and Management, vol. 53, pp. 337-345, Jan. 2012.

[27] W. Nwesaty, A. I. Bratcu, and O. Sename, "Optimal frequency separation of power sources by multivariable $l p v / h_{\infty}$; control: Application to on-board energy management systems of electric vehicles," in Proceedings of 2014 IEEE 53rd Annual Conference on Decision and Control - CDC 2014, pp. 5636-5641, Dec 2014.

[28] P. Apkarian, P. Gahinet, and G. Becker, "Self-scheduled $\mathcal{H}_{\infty}$ control of linear parameter-varying systems: a design example," Automatica, vol. 31, pp. 12511261, Sept. 1995.

[29] D. Goldberg and J. Holland, "Genetic Algorithms and Machine Learning," Machine Learning, vol. 3, no. 2-3, pp. 95-99, 1988.

[30] E. Zitzler, M. Laumanns, and L. Thiele, SPEA2: Improving the strength Pareto evolutionary algorithm. Eidgenössische Technische Hochschule Zürich (ETH), Institut für Technische Informatik und Kommunikationsnetze (TIK), 2001.

[31] C. Scherer, P. Gahinet, and M. Chilali, "Multiobjective output-feedback control via LMI optimization," IEEE Transactions on Automatic Control, vol. 42, pp. 896-911, July 1997.

[32] A. Do, O. Sename, L. Dugard, and others, "LPV modelling and control of semiactive dampers in automotive systems," Control of Linear Parameter Varying Systems with Applications, 2012.

[33] C. Poussot-Vassal and P. Vuillemin, "Introduction to MORE: A MOdel REduction toolbox," in 2012 IEEE International Conference on Control Applications (CCA), pp. 776-781, Oct. 2012. 